\title{
A generalized approach to the design of variable fractional-delay FIR digital filters
}

\author{
Jong-Jy Shyu ${ }^{\mathrm{a}, *}$, Soo-Chang Pei ${ }^{\mathrm{b}}$ \\ ${ }^{a}$ Department of Electrical Engineering, National University of Kaohsiung, Taiwan, ROC \\ ${ }^{\mathrm{b}}$ Department of Electrical Engineering, National Taiwan University, Taiwan, ROC
}

Received 4 September 2007; received in revised form 15 November 2007; accepted 6 December 2007

Available online 23 December 2007

\begin{abstract}
In this paper, a complex-oriented weighted least-squares approach is proposed for the design of arbitrary variable fractional-delay FIR filters. The objective error function is formulated in a quadratic form, such that the filter coefficients can be obtained by proper matrix/vector operation, including matrix inversion. However, all elements of related matrices and vectors can be represented in closed forms, and the matrix to be inversed is a positive-definite Hermitian symmetric matrix, which can be decomposed by the Cholesky factorization, such that computation time can be effectively reduced and the ill condition can be avoided. Comparing with the existing methods, the proposed method can be applied to design arbitrary complex coefficient or real coefficient variable fractional-delay filters. Several examples are presented to demonstrate the flexibility and effectiveness of the proposed method.
\end{abstract}

(C) 2007 Elsevier B.V. All rights reserved.

Keywords: Variable fractional-delay FIR filter; Weighted least-squares approach; FIR filter; Cholesky factorization; Partial-band differentiator

\section{Introduction}

In this paper, a complex-oriented weighted leastsquares approach is proposed to design arbitrary complex coefficient variable fractional-delay (VFD) FIR filters, and the design of real coefficient filters is just a special case of them. Recently, there are several papers [1-11] concerning the design of real coefficient VFD filters, which are widely used in discrete-time signal interpolation, timing offset recovery in digital receivers, speech coding and synthesis and comb filter

\footnotetext{
*Corresponding author.

E-mail addresses: jshyu@nuk.edu.tw (J.-J. Shyu), pei@cc.ee.ntu.edu.tw (S.-C. Pei).
}

design, etc. [12-16]. As to the implementation of such filters, the Farrow structure is the most popular one $[17,18]$. Over the past decade, the design of complex coefficient filters has also received considerable attention [19-22]. Among them, Ref. [19] deals with the design of complex coefficient variable digital filters, in which the successive vector-array decomposition method is applied. In this paper, the conventional weighted least-squares approach is extended to complex-oriented approximation method, which can be used to design arbitrary variable fractional-delay filters, including both complex coefficient and real coefficient filters, with no need for iteration.

This paper is organized as follows. Section 2 introduces the derivation of problem formulation. It can 
be found that the filter coefficients can be obtained by evaluating matrix/vector operation including matrix inversion. Especially, to avoid ill condition when matrix inversion is to be found, the related matrix is represented in a positive-definite Hermitian symmetric matrix, which can be decomposed by the Cholesky factorization process. To demonstrate the effectiveness of the proposed method, several numerical VFD examples including a non-symmetric singlepassband filter, a partial-band differentiator, and a non-symmetric pure delay filter are presented in Section 3. It is noted that all elements of matrices or vectors can be represented in closed forms, such that the computation time can be effectively reduced. Moreover, the design of symmetric pure VFD filters is also given in Section 3, which demonstrates the flexibility of the proposed method. Finally, the conclusions are given in Section 4.

\section{Problem formulation}

In this paper, the desired variable fractional-delay frequency response is given by

$$
\begin{aligned}
& H_{\mathrm{d}}(\omega, p) \\
& \quad= \begin{cases}M(\omega) \mathrm{e}^{-\mathrm{j} \omega p}, & \omega \in \text { passbands, } \\
0, & \omega \in \text { stopbands, }\end{cases}
\end{aligned}
$$

where $M(\omega)$ is the desired pure real or pure imaginary amplitude response, and the variable transfer function is characterized by

$H(z, p)=\sum_{n=-N}^{N} h_{n}(p) z^{-n}$,

where the coefficients $h_{n}(p)$ s are generally expressed as the polynomials of $p$ as

$h_{n}(p)=\sum_{m=0}^{M} a(n, m) p^{m}$,

in which $a(n, m)$ s are complex-valued coefficients. So, the frequency response of the designed system can be represented as

$H\left(\mathrm{e}^{\mathrm{j} \omega}, p\right)=\sum_{n=-N}^{N} \sum_{m=0}^{M} a(n, m) p^{m} \mathrm{e}^{-\mathrm{j} n \omega}$.

Define

$$
\mathbf{A}=\left[\begin{array}{llll}
\mathbf{A}_{-N}^{\mathrm{T}} & \mathbf{A}_{-N+1}^{\mathrm{T}} & \cdots & \mathbf{A}_{N}^{\mathrm{T}}
\end{array}\right]^{\mathrm{T}}
$$

and

$$
\mathbf{E}(\omega, p)=\left[\begin{array}{llll}
\mathbf{E}_{-N}^{\mathrm{T}}(\omega, p) & \mathbf{E}_{-N+1}^{\mathrm{T}}(\omega, p) & \cdots & \mathbf{E}_{N}^{\mathrm{T}}(\omega, p)
\end{array}\right]^{\mathrm{T}},
$$

where $\mathrm{T}$ denotes transpose operator,

$$
\begin{aligned}
\mathbf{A}_{n}= & {\left[\begin{array}{llll}
a(n, 0) & a(n, 1) & \cdots & a(n, M)
\end{array}\right]^{\mathrm{T}}, } \\
& -N \leqslant n \leqslant N
\end{aligned}
$$

and

$$
\begin{aligned}
\mathbf{E}_{n}(\omega, p)= & {\left[\begin{array}{llll}
\mathrm{e}^{-\mathrm{j} n \omega} & p \mathrm{e}^{-\mathrm{j} n \omega} & \cdots & p^{M} \mathrm{e}^{-\mathrm{j} n \omega}
\end{array}\right]^{\mathrm{T}}, } \\
& -N \leqslant n \leqslant N,
\end{aligned}
$$

then Eq. (4) can be rewritten in vector product form as

$$
H\left(\mathrm{e}^{\mathrm{j} \omega}, p\right)=\mathbf{A}^{\mathrm{T}} \mathbf{E}(\omega, p)=\mathbf{E}^{\mathrm{T}}(\omega, p) \mathbf{A} .
$$

In order to approach $H_{\mathrm{d}}(\omega, p)$, the objective error function is defined by

$$
\begin{aligned}
e(\mathbf{A})= & \int_{R} \int_{p_{1}}^{p_{2}} W(\omega, p)\left|H_{\mathrm{d}}(\omega, p)-H\left(\mathrm{e}^{\mathrm{j} \omega}, p\right)\right|^{2} \mathrm{~d} p \mathrm{~d} \omega \\
= & \int_{R} \int_{p_{1}}^{p_{2}} W(\omega, p)\left[H_{\mathrm{d}}^{*}(\omega, p)-H^{*}\left(\mathrm{e}^{\mathrm{j} \omega}, p\right)\right] \\
& \times\left[H_{\mathrm{d}}(\omega, p)-H\left(\mathrm{e}^{\mathrm{j} \omega}, p\right)\right] \mathrm{d} p \mathrm{~d} \omega \\
= & \int_{R} \int_{p_{1}}^{p_{2}} W(\omega, p)\left[H_{\mathrm{d}}^{*}(\omega, p) H_{\mathrm{d}}(\omega, p)\right. \\
& -H_{\mathrm{d}}^{*}(\omega, p) H\left(\mathrm{e}^{\mathrm{j} \omega}, p\right)-H_{\mathrm{d}}(\omega, p) H^{*}\left(\mathrm{e}^{\mathrm{j} \omega}, p\right) \\
& \left.+H^{*}\left(\mathrm{e}^{\mathrm{j} \omega}, p\right) H\left(\mathrm{e}^{\mathrm{j} \omega}, p\right)\right] \mathrm{d} p \mathrm{~d} \omega \\
= & s+\mathbf{A}^{\mathrm{T}} \mathbf{P}+\mathbf{A}^{\mathrm{H}} \mathbf{P}^{*}+\mathbf{A}^{\mathrm{H}} \mathbf{Q} \mathbf{A}
\end{aligned}
$$

where $W(\omega, p)$ is a positive-valued weighting function, $R$ represents the region of passbands and stopbands, * denotes complex-conjugate operator, $H$ denotes Hermitian transpose operator,

$s=\int_{R} \int_{p_{1}}^{p_{2}} W(\omega, p) H_{\mathrm{d}}^{*}(\omega, p) H_{\mathrm{d}}(\omega, p) \mathrm{d} p \mathrm{~d} \omega$,

$\mathbf{P}=-\int_{R} \int_{p_{1}}^{p_{2}} W(\omega, p) H_{\mathrm{d}}^{*}(\omega, p) \mathbf{E}(\omega, p) \mathrm{d} p \mathrm{~d} \omega$

and

$\mathbf{Q}=\int_{R} \int_{p_{1}}^{p_{2}} W(\omega, p) \mathbf{E}^{*}(\omega, p) \mathbf{E}^{\mathrm{T}}(\omega, p) \mathrm{d} p \mathrm{~d} \omega$.

To minimize e(A), Eq. (7) is differentiated with respect to the unknown vector $\mathbf{A}$ and set the result 
to zero as:

$\frac{\partial e(\mathbf{A})}{\partial \mathbf{A}}=2 \mathbf{P}^{*}+2 \mathbf{Q A}=\mathbf{0}$,

so the optimal coefficient vector in weighted leastsquares sense is given by

$\mathbf{A}=-\mathbf{Q}^{-1} \mathbf{P}^{*}$

and the minimal error $e_{\min }$ can be obtained by substituting Eq. (10) into Eq. (7). Notice that $\mathbf{Q}$ is a positive-definite Hermitian symmetric matrix, which can be decomposed as

$\mathbf{Q}=\mathbf{X}^{\mathrm{H}} \mathbf{X}$

by using the Cholesky factorization, such that the ill-conditioned problem which occurs when $\mathbf{Q}^{-1}$ is to be found can be avoided. Once $\mathbf{A}$ is found, the filter coefficients $a(n, m)$ s can be obtained easily by Eq. (5a).

\section{Numerical examples and discussions}

To demonstrate the effectiveness of the proposed method, several examples are presented in this section. For simplicity, $W(\omega, p)=1$ is used in this paper. To evaluate the performance, the normalized root mean squared error $\varepsilon_{2}$ and the maximum absolute group-delay error $\varepsilon_{\tau}$ are defined by

$$
\begin{aligned}
\varepsilon_{2} & =\left[\frac{\int_{R} \int_{p_{1}}^{p_{2}}\left|H_{\mathrm{d}}(\omega, p)-H\left(\mathrm{e}^{\mathrm{j} \omega}, p\right)\right|^{2} \mathrm{~d} p \mathrm{~d} \omega}{\int_{R} \int_{p_{2}}^{p_{2}}\left|H_{\mathrm{d}}(\omega, p)\right|^{2} \mathrm{~d} p \mathrm{~d} \omega}\right]^{1 / 2} \times 100 \% \\
& =\left[\frac{s+\mathbf{A}^{\mathrm{T}} \mathbf{P}+\mathbf{A}^{\mathrm{H}} \mathbf{P}^{*}+\mathbf{A}^{H} \mathbf{Q A}}{s}\right]^{1 / 2} \times 100 \%
\end{aligned}
$$

To compute $\varepsilon_{\tau}$, the frequency $\omega$ and the fractionaldelay $p$ are uniformly sampled at the step sizes $(2 \pi /$ $1000)$ and $\left(\left(p_{2}-p_{1}\right) / 40\right)$, respectively.

Example 1. In this example, a single-passband nonsymmetric variable fractional-delay filter is designed with the desired response

$H_{\mathrm{d}}(\omega, p)= \begin{cases}\mathrm{e}^{-\mathrm{j} \omega p}, & \omega \in\left(\omega_{p_{1}}, \omega_{p_{2}}\right) \text { and } p \in\left(p_{1}, p_{2}\right), \\ 0, & \omega \in\left(-\pi, \omega_{s_{1}}\right) \cup\left(\omega_{s_{2}}, \pi\right) .\end{cases}$

The elements of vector $\mathbf{P}$ are given by

$$
\begin{aligned}
\mathbf{P}(i) & =-\int_{\omega_{p_{1}}}^{\omega_{p_{2}}} \int_{p_{1}}^{p_{2}} \mathrm{e}^{\mathrm{j} \omega p} \mathrm{e}^{-\mathrm{j} n \omega} p^{m} \mathrm{~d} p \mathrm{~d} \omega, \\
0 & \leqslant i \leqslant(2 N+1)(M+1)-1, \\
n & =\left\lfloor\frac{i}{M+1}\right\rfloor-N, \quad m=\bmod (i, M+1),
\end{aligned}
$$

while the elements of matrix $\mathbf{Q}$ are given by

$$
\begin{aligned}
& \mathbf{Q}(i, l)= \int_{\omega_{p_{1}}}^{\omega_{p_{2}}} \int_{p_{1}}^{p_{2}}\left(\mathrm{e}^{-\mathrm{j} n \omega} p^{m}\right)^{*}\left(\mathrm{e}^{-\mathrm{j} \hat{n} \omega} p^{\hat{m}}\right) \mathrm{d} p \mathrm{~d} \omega \\
& \quad+\int_{\omega_{s_{2}}}^{\omega_{s_{3}}} \int_{p_{1}}^{p_{2}}\left(\mathrm{e}^{-\mathrm{j} n \omega} p^{m}\right)^{*}\left(\mathrm{e}^{-\mathrm{j} \hat{n} \omega} p^{\hat{m}}\right) \mathrm{d} p \mathrm{~d} \omega, \\
& 0 \leqslant i, l \leqslant(2 N+1)(M+1)-1, \\
& n=\left\lfloor\frac{i}{M}\right\rfloor-N, \quad m=\bmod (i, M+1), \\
& \hat{n}=\left\lfloor\frac{l}{M}\right\rfloor-N, \quad \hat{m}=\bmod (l, M+1),
\end{aligned}
$$

where $\omega_{s_{3}}=\omega_{s_{1}}+2 \pi$. Eq. (15b) can be easily represented in closed-forms as

$$
\mathbf{Q}(i, l)= \begin{cases}\frac{p_{2}^{m+\hat{m}+1}-p_{1}^{m+\hat{m}+1}}{m+\hat{m}+1}\left(\omega_{p_{2}}-\omega_{p_{1}}+\omega_{s_{3}}-\omega_{s_{2}}\right), & \text { if } n=\hat{n}, \\ \frac{p_{2}^{m+\hat{m}+1}-p_{1}^{m+\hat{m}+1}}{m+\hat{m}+1} \times \frac{\mathrm{e}^{\mathrm{j}(n-\hat{n}) \omega_{p_{2}}}-\mathrm{e}^{\mathrm{j}(n-\hat{n}) \omega_{p_{1}}+\mathrm{e}^{\mathrm{j}(n-\hat{n}) \omega_{s_{3}}}-\mathrm{e}^{\mathrm{j}(n-\hat{n}) \omega_{s_{2}}}},}{j(n-\hat{n})}, \text { otherwise }\end{cases}
$$

and

$\varepsilon_{\tau}=\max \left\{|\tau(\omega, p)-p|, \quad \omega \in\right.$ passbands, $\left.p \in\left(p_{1}, p_{2}\right)\right\}$

respectively, where $\tau(\omega, p)$ is the actual fractional group-delay of the designed filter defined by

$\tau(\omega, p)=-\frac{\mathrm{d}}{\mathrm{d} \omega} \arg \left(H\left(\mathrm{e}^{\mathrm{j} \omega}, p\right)\right)$. but it is difficult to get the closed-forms of Eq. (15a). However, $\mathbf{P}(i)$ can be evaluated and approximated by applying the Taylor series expansion of $\mathrm{e}^{\mathrm{j} w p}$ as:

$$
\begin{aligned}
\mathbf{P}(i) & =-\int_{\omega_{p_{1}}}^{\omega_{p_{2}}} \int_{p_{1}}^{p_{2}} \sum_{k=0}^{\infty} \frac{(\mathrm{j} \omega p)^{k}}{k !} \mathrm{e}^{-\mathrm{j} n \omega} p^{m} \mathrm{~d} p \mathrm{~d} \omega \\
& \cong \sum_{k=0}^{K} \frac{-\mathrm{j}^{k}}{k !}\left(\int_{\omega_{p_{1}}}^{\omega_{p_{2}}} \omega^{k} \mathrm{e}^{-\mathrm{j} n \omega} \mathrm{d} \omega\right)\left(\int_{p_{1}}^{p_{2}} p^{k} p^{m} \mathrm{~d} p\right)
\end{aligned}
$$


a
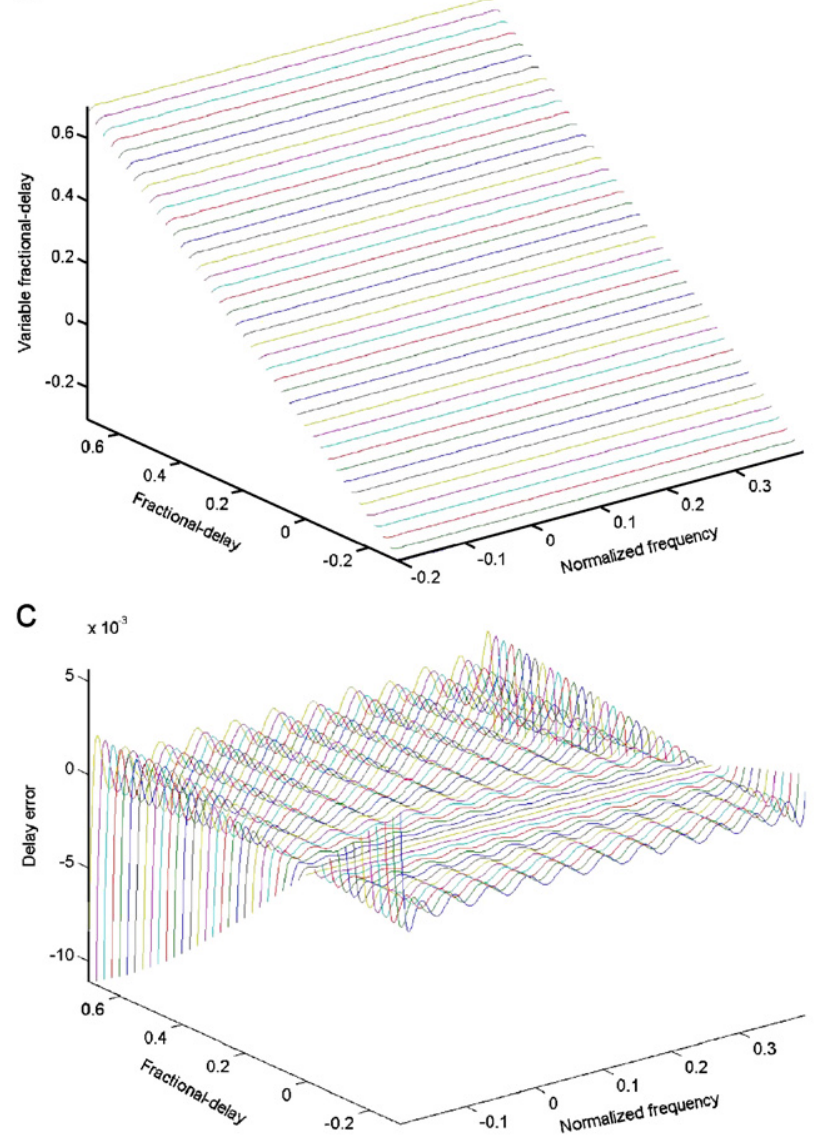

b

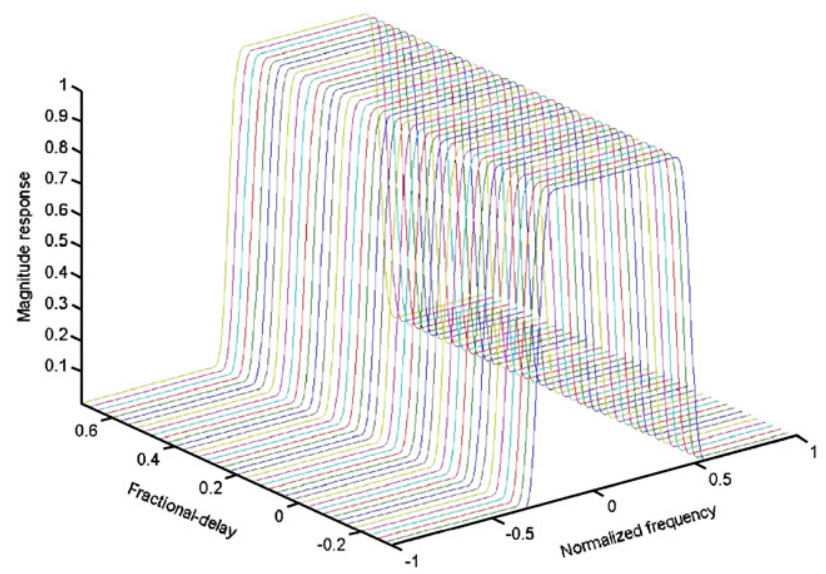

d

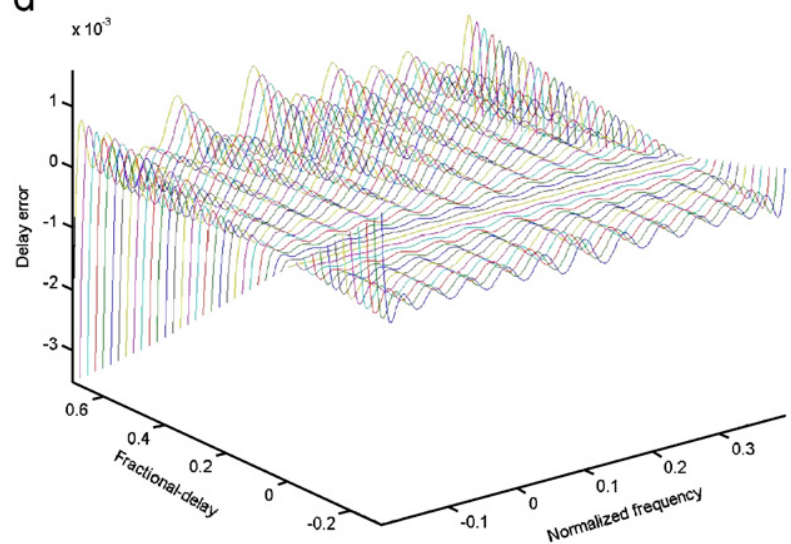

Fig. 1. Example 1: Design of a single-passband non-symmetric variable fractional-delay filter; (a) variable fractional-delay response in passband, (b) magnitude response, (c) delay error with uniform weighting function, and (d) delay error with non-uniform weighting function.

$$
\begin{aligned}
= & \sum_{k=0}^{K} \frac{-\mathrm{j}^{k}}{k !} \frac{p_{2}^{k+m+1}-p_{1}^{k+m+1}}{k+m+1} \\
& \times\left[\int_{\omega_{p_{1}}}^{\omega_{p_{2}}} \omega^{k} \cos (n \omega) \mathrm{d} \omega-\mathrm{j} \int_{\omega_{p_{1}}}^{\omega_{p_{2}}} \omega^{k} \sin (n \omega) \mathrm{d} \omega\right]
\end{aligned}
$$

and the closed-forms can be obtained easily by integration formulas as in Ref. [1]. In this paper, $K=20$ is used, and the result is satisfactory. For example, when $N=33, M=7, \omega_{s_{1}}=-0.35 \pi, \omega_{p_{1}}=$ $-0.2 \pi, \omega_{p_{2}}=0.4 \pi, \omega_{s_{2}}=0.55 \pi, p_{1}=-0.3, p_{2}=0.7$, the actual variable fractional-delay response, magnitude response and delay error are presented in Fig. 1(a), (b) and (c), respectively, and $\varepsilon_{2}=0.0042454 \%, \varepsilon_{\tau}=0.0112$. For improving the maximum absolute group-delay $\varepsilon_{\tau}$, a non-uniform weighting function can be used. For example, let

$$
W(\omega, p)= \begin{cases}1+20\left|\omega-\frac{\omega_{p_{1}}+\omega_{p_{2}}}{2}\right|, & \omega \in \text { passband, } \\ 1, & \omega \in \text { stopbands }\end{cases}
$$

the delay error is presented in Fig. 1(d) and $\varepsilon_{\tau}=0.0035$.

Example 2. In this example, a partial-band variable fractional-delay differentiator is designed and the desired response is given by

$H_{\mathrm{d}}(\omega, p)= \begin{cases}\mathrm{j} \omega \mathrm{e}^{-\mathrm{j} \omega p}, & \omega \in\left(\omega_{p_{1}}, \omega_{p_{2}}\right), p \in\left(p_{1}, p_{2}\right), \\ 0, & \omega \in\left(-\pi, \omega_{s_{1}}\right) \cup\left(\omega_{s_{2}}, \pi\right),\end{cases}$

in which $0<\omega_{s_{1}}<\omega_{p_{1}}<\omega_{p_{2}}<\omega_{s_{2}} \leqslant \pi$. Also when $W=(\omega, p)=1$, the elements of matrix $\mathbf{Q}$ are the same as Eq. (16a), and the elements of vector $\mathbf{P}$ can 


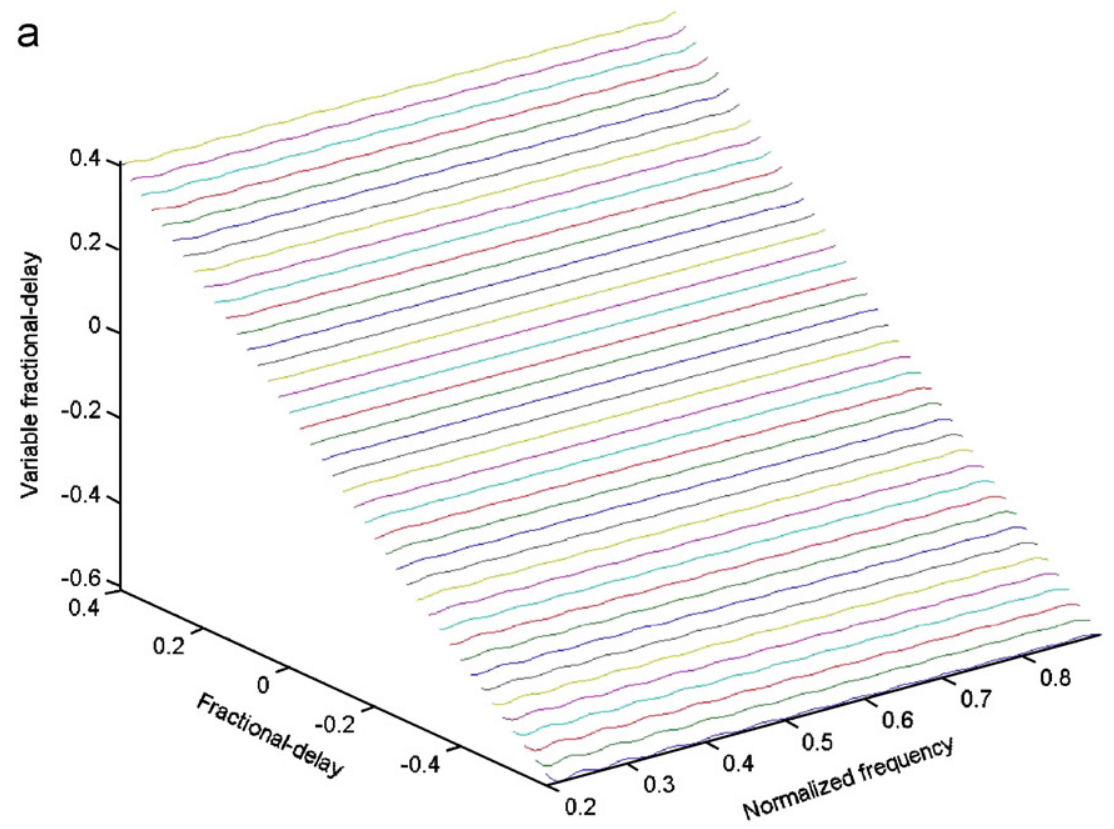

b

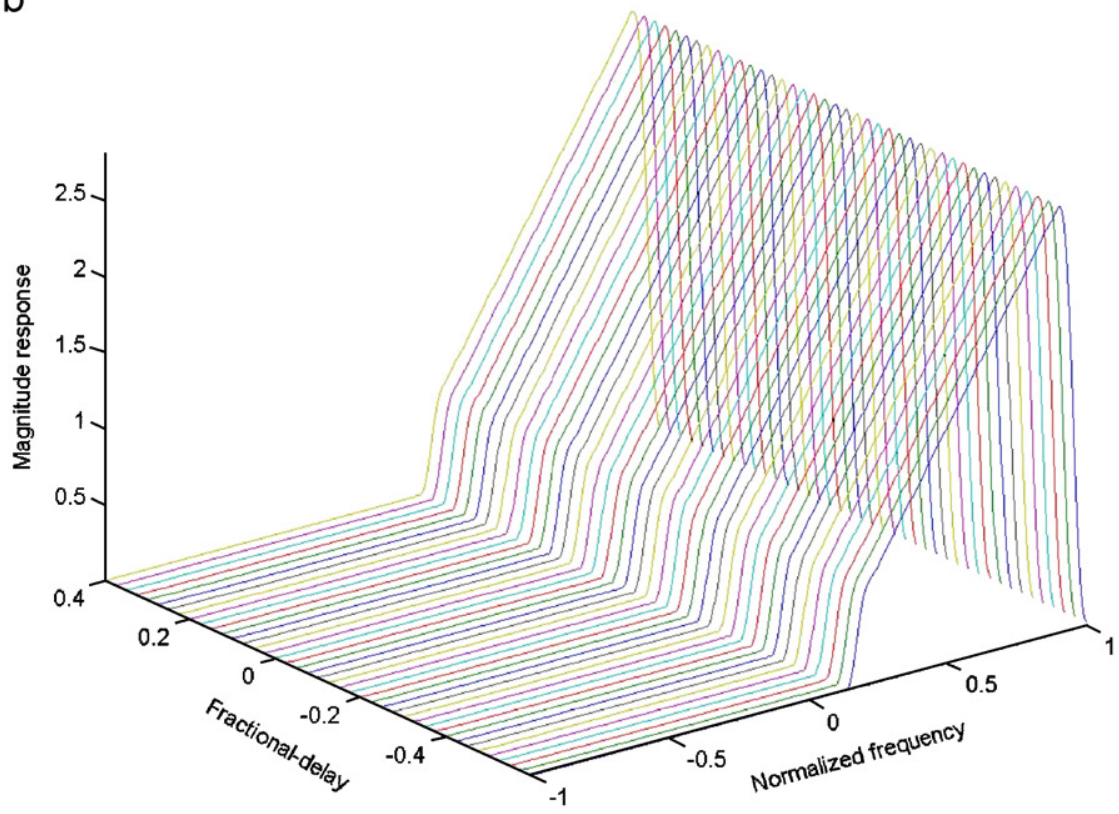

Fig. 2. Example 2: Design of a partial-band variable fractional-delay differentiator; (a) variable fractional-delay response in passband and (b) magnitude response.

be represented as follows:

$$
\begin{aligned}
\mathbf{P}(i) \cong & \sum_{k=0}^{K} \frac{\mathrm{j}^{k+1}}{k !} \frac{p_{2}^{k+m+1}-p_{1}^{k+m+1}}{k+m+1} \\
& \times\left[\int_{\omega_{p_{1}}}^{\omega_{p_{2}}} \omega^{k+1} \cos (n \omega) \mathrm{d} \omega-\mathrm{j} \int_{\omega_{p_{1}}}^{\omega_{p_{2}}} \omega^{k+1} \sin (n \omega) \mathrm{d} \omega\right]
\end{aligned}
$$

$$
\begin{aligned}
& 0 \leqslant 2 i \leqslant(2 N+1)(M+1)-1, \\
& n=\left\lfloor\frac{i}{M+1}\right\rfloor-N, \quad m=\bmod (i, M+1),
\end{aligned}
$$

which can be formulated into closed-forms after some mathematical manipulation. Fig. 2(a) and (b) illustrates the obtained variable fractional-delay response and magnitude response, respectively, when 

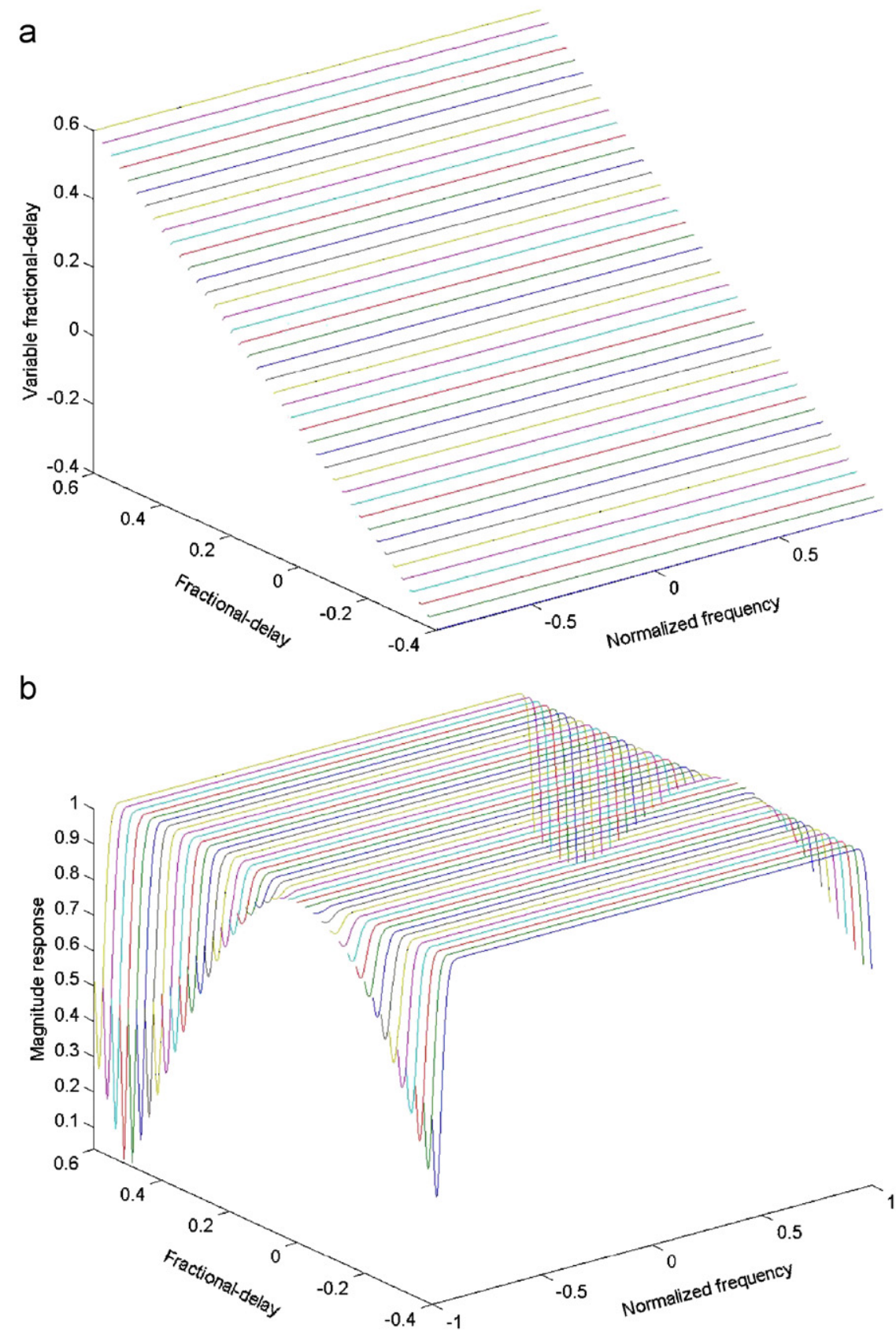

Fig. 3. Example 3: Design of a pure variable fractional-delay filter; (a) variable fractional-delay response and (b) magnitude response.

$N=33, M=7, \omega_{s_{1}}=0.14 \pi, \omega_{p_{1}}=0.2 \pi, \omega_{p_{2}}=0.9 \pi$, $\omega_{s_{2}}=\pi, p_{1}=-0.6, p_{2}=0.4$ are used. The obtained normalized root mean squared error $\varepsilon_{2}=0.12 \%$, and the maximum absolute group-delay error $\varepsilon_{\tau}=0.0157$.

Example 3. An $N=33, M=7$ pure variable fractional-delay system is designed in this example, and the desired frequency response is given by

$$
\begin{aligned}
& H_{\mathrm{d}}(\omega, p)=\mathrm{e}^{-\mathrm{j} \omega p}, \quad\left(\omega_{p_{1}}=-0.88 \pi\right)<\omega<\left(\omega_{p_{2}}=0.92 \pi\right), \\
& \quad\left(p_{1}=-0.4\right)<p<\left(p_{2}=0.6\right) .
\end{aligned}
$$

The obtained variable fractional-delay response and magnitude response are presented in Fig. 3(a) and (b) 
respectively, which result in $\varepsilon_{2}=0.0016844 \%$ and $\varepsilon_{\tau}=0.0038$. For symmetric pure variable fractionaldelay filter design with $\omega_{p 1}=-\omega_{p 2}$ and $p_{1}=-p_{2}$, it is found that the filter coefficients are real, which also reveal the symmetric property

$a(-n, m)=(-1)^{m} a(n, m), \quad 0 \leqslant n \leqslant N, 1 \leqslant m \leqslant M$

and

$a(n, 0)=\delta(n), \quad-N \leqslant n \leqslant N$,

as shown in Ref. [1]. For example, when $N=33$, $M=7, \omega_{p_{1}}=-\omega_{p_{2}}=-0.9 \pi, p_{1}=-p_{2}=-0.5$, the obtained $\varepsilon_{2}=0.00028753 \%$ and $\varepsilon_{\tau}=0.0038$. Comparing with those obtained in Ref. [1] in which $\varepsilon_{2}=0.000257 \%$ and $\varepsilon_{\tau}=0.001863$, although the performance of the proposed method is not so good, but our performance is much better than that in Ref. [2] in which the variable fractional-delay filter is designed with $N=20, M=5, \omega_{p_{1}}=-\omega_{p_{2}}=$ $-0.9 \pi, p_{1}=-p_{2}=-0.5$ and the root mean squared error $\varepsilon_{\mathrm{rms}}=0.0038$ in Ref. [2], while $\varepsilon_{\mathrm{rms}}=$ 0.00025489 by the proposed method, where $\varepsilon_{\mathrm{rms}}$ is defined by

$\varepsilon_{\text {rms }}=\left[\int_{0}^{0.9 \pi} \int_{-0.5}^{0.5}\left|H_{\mathrm{d}}(\omega, p)-H\left(\mathrm{e}^{\mathrm{j} \omega}, p\right)\right|^{2} \mathrm{~d} p \mathrm{~d} \omega\right]^{1 / 2}$.

From the above examples, it is found that the proposed method can deal with the variable design with arbitrary delay range that depends on the applications. Also, when the dimension of $\mathbf{Q}$ is too large, the ill-conditioned problem will occur. For instance, it will appear when $N=33$ is used in Example 3, but it does not occur when $N=20$ is used. However, the ill-conditioned problem can be easily avoided by using the Cholesky factorization, which is discussed in Section 2.

\section{Conclusions}

In this paper, the conventional weighted leastsquares method has successfully been extended to complex-oriented approach such that it can design arbitrary variable fractional-delay FIR filters, including real coefficient and complex coefficient filters. Generally, all elements in related matrices or vectors can be represented in closed forms, and the nearly optimal solutions can be obtained. Also, the proposed method can be easily extended to design other types of variable filters or multidimensional variable filters.

\section{References}

[1] T.-B. Deng, Y. Lian, Weighted-least-squares design of variable fractional-delay FIR filters using coefficient symmetry, IEEE Trans. Signal Process. 54 (8) (2006) 3023-3038.

[2] C.-C. Tseng, Eigenfilter approach for the design of variable fractional delay FIR and all-pass filters, IEEE Proc. Vis. Image Signal Process. 149 (5) (2002) 297-303.

[3] C.-C. Tseng, Design of fractional delay filter, Nyquist filter, lowpass filter and diamond-shaped filter, Signal Process. 87 (2007) 584-601.

[4] T.-B. Deng, Closed-form design and efficient implementation of variable digital filters with simultaneously tunable magnitude and fractional delay, IEEE Trans. Signal Process. 52 (6) (2004) 1668-1681.

[5] W.-S. Lu, T.-B. Deng, An improved weighted least-squares design for variable fractional delay FIR filters, IEEE Trans. Circuits Syst. I, Analog Digit. Signal Process. 46 (8) (1999) 1035-1040.

[6] T.-B. Deng, Y. Nakagawa, SVD-based design and new structure for variable fractional-delay digital filters, IEEE Trans. Signal Process. 52 (9) (2004) 2513-2527.

[7] H. Zhao, J. Yu, A simple and efficient design of variable fractional-delay FIR filters, IEEE Trans. Circuits Syst. II, Express Brief. 53 (2) (2006) 157-160.

[8] S.-C. Pei, P.-H. Wang, H.-S. Lin, Closed-form design of maximally flat FIR fractional-delay filter, IEEE Signal Process. Lett. 13 (7) (2006) 405-408.

[9] T.I. Laakso, V. Valimaki, M. Karjalainen, U.K. Laine, Splitting tools for fractional delay filter design, IEEE Signal Process. Mag. 13 (1) (1996) 30-60.

[10] T.-B. Deng, Symmetry-based low-complexity variable fractional-delay FIR filters, in: Proceedings of the 2004 IEEE International Symposium on Communications Information Technologies (ISCIT'04), October 2004, pp. 194-199.

[11] C.-C. Tseng, Design of variable fractional delay FIR filters using symmetry, in: Proceedings of the 2004 IEEE International Symposium on Circuits and Systems, May 2004, pp. $477-480$.

[12] H. Johansson, P. LÖwenborg, Reconstruction of nonuniformly sampled bandlimited signals by means of digital fractional delay filters, IEEE Trans. Signal Process. 50 (11) (2002) 2757-2767.

[13] G.-S. Liu, C.-W. Wei, A new variable fractional sample delay filter with nonlinear interpolation, IEEE Trans. Circuits Syst. II, Analog Digit. Signal Process. 39 (2) (1992) 123-126.

[14] F.M. Gardner, Interpolation in digital modems - Part I: Fundamentals, IEEE Trans. Commun. 41 (3) (1993) 502-508

[15] K. Rajamani, Y.S. Lai, C.W. Farrow, An efficient algorithm for sample rate conversion from CD to DAT, IEEE Signal Process. Lett. 7 (10) (2000) 288-290. 
[16] S.-C. Pei, C.-C. Tseng, A comb filter design using fractional sample delay, IEEE Trans. Circuits Syst. II, Analog Digit. Signal Process. 45 (6) (1998) 635-649.

[17] C.W. Farrow, A continuously variable digital delay element, in: Proceedings of the ISCAS, 1988, pp. 2641-2645.

[18] C.K.S. Pun, Y.C. Wu, S.C. Chan, K.L. Ho, On the design and efficient implementation of the Farrow structure, IEEE Signal Process. Lett. 10 (7) (2003) 189-192.

[19] T.-B. Deng, Design of complex-coefficient variable digital filters using successive vector-array decomposition, IEEE Trans. Circuits Syst. I, Reg. Papers, 52 (5) (2005) 932-942.
[20] Z. Lin, Y. Liu, Design of complex FIR filters with reduced group delay error using semidefinite programming, IEEE Signal Process. Lett. 13 (9) (2006) 529-532.

[21] S.C. Chan, K.M. Tsui, On the design of real and complex FIR filters with flatness and peak error constraints using semidefinite programmimg, in: Proceedings of the IEEE International Symposium on Circuits Systems, May 2004, vol. III, pp. 125-128.

[22] J.-J. Shyu, S.-C. Pei, K.-C. Fu, Complex Lagrange multiplier approach to the design of arbitrary complex coefficient FIR digital filters, Signal Process. 35 (1994) 117-130. 